\title{
Renormalization approach to the self-organized critical behavior of sandpile models
}

\author{
Alessandro Vespignani, ${ }^{1,2}$ Stefano Zapperi, ${ }^{1,3}$ and Luciano Pietronero ${ }^{1}$ \\ ${ }^{1}$ Dipartimento di Fisica, Università di Roma "La Sapienza," Piazzale Aldo Moro 2, 00185 Roma, Italy \\ ${ }^{2}$ Department of Mathematics, Yale University, Box 208283, New Haven, Connecticut 06520-8283 \\ ${ }^{3}$ Center for Polymer Studies and Department of Physics, Boston University, Boston, Massachusetts 02215
}

(Received 2 August 1994)

\begin{abstract}
We introduce a renormalization scheme of a type that is able to describe the self-organized critical state (SOC) of sandpile models. We have defined a characterization of the phase space that allows us to study the evolution of the dynamics under change of scale. In addition, a stationarity condition provides a feedback mechanism that drives the system to its critical state. We obtain an attractive fixed point in the phase space of the parameters that clarifies the self-organized critical nature of these systems. The universality class of several models is identified by studying the properties of the basin of attraction of this fixed point. We compute analytically the avalanche exponent $\tau$ and the dynamical exponent $z$ for sandpile models in $d=2$. The values obtained are in very good agreement with computer simulations. The renormalization scheme can also be applied to study nonconservative sandpile models. The result is that the introduction of a dissipation parameter destroys the critical properties as suggested from simulations. The present theoretical framework seems particularly suitable for all SOC problems and can be naturally extended to other systems showing a critical nonequilibrium stationary state.
\end{abstract}

PACS number(s): 64.60.Ak, 02.50. $-\mathrm{r}, 05.40 .+\mathrm{j}$

\section{INTRODUCTION}

The concept of self-organized criticality (SOC) has been invoked by Bak, Tang, and Wiesenfeld [1] as a possible unifying framework to describe a vast class of dynamically driven systems which evolve spontaneously toward a critical stationary state with no characteristic time or length scale. The name self-organized criticality comes from the fact that, unlike phase transitions in equilibrium statistical physics, the critical state is reached without the need to fine tune any control parameter; i.e., the critical state is an attractor of the dynamics. To illustrate the basic ideas of SOC, Bak, Tang, and Wiesenfeld used a cellular automaton inspired by the flow of avalanches in a pile of sand. In these models the sand is added grain by grain on a $d$-dimensional lattice until unstable sand (too large local slope of the pile) slides off. In this way the pile reaches a stationary critical state, characterized by a critical slope, in which additional grains of sand will fall off the pile via avalances that can be small or cover the entire size of the system. The critical state is characterized by a power law for the size and lifetime distribution of avalanches which therefore do not have a characteristic length scale. This class of automaton can be used to model many avalanche phenomena, interpreting the sand as energy, mechanical stress, heat memory, etc. One can add more examples from other fields like economy, social sciences and biology, as well [2].

Other examples of SOC behavior can be found in fractal growth phenomena, such as diffusion limited aggregation (DLA) [3,4]. These models lead spontaneously to a statistically stationary state where a self-similar pattern is generated. Also in this case the critical state is an attractor for the dynamics, and it does not require any tuning parameter.
The general conditions under which a physical system shows SOC properties have recently been discussed $[5,6]$. For a sandpile these conditions are identified in a stationary state characterized by a diffusive dynamics that satisfies a global conservation law and the existence of a small scale rigidity, i.e., the critical threshold, for the microscopic dynamics [5]. From the perspective of usual critical phenomena this new situation can be described as a feedback mechanism of the order parameter into the control parameter [6]. However, despite their simple definitions, both SOC models and DLA are characterized by a nonlinear dynamics that involves complex processes: either an entire random walk or a whole avalanche. In fact, in SOC phenomena one deals with open nonlinear systems in which the temporal evolution cannot be eliminated in view of ergodicity and has to be considered explicitly. For these reasons, the application of usual theoretical methods to describe the critical state and to the calculation of the critical exponents turns out to be very problematic. This leads various authors $[7,8]$ to believe that both DLA and sandpile models pose a new type of problem for which it would be desirable to define a common theoretical framework.

For DLA-like problems we have introduced a theoretical approach named the fixed scale transformation (FST) [9] that is based on two steps: one is the study of correlation properties at a given scale generated asymptotically by the dynamical evolution, and the second is the identification of scale-invariant dynamics. This second step corresponds essentially to a renormalization group (RG) scheme for the dynamics [10], while the first step allows us to relate the fixed-point properties of the dynamics directly to the critical exponents. In this paper we follow a similar reasoning to develop an alternative type of renormalization group scheme for the dynamics of SOC 
models [11], and we show its application to the class of the critical height sandpile automata. The first step of the method is the identification of the parameters that characterize the stationary and dynamical properties of the critical state. This corresponds to the identification of the proper phase space in which to study the evolution of the dynamics under scale transformation. We then find the renormalization equations that link the parameters at a generic scale $2 b$ with those at scale $b$, and we couple them with the energy balance equation which gives the stationarity condition of the system. By iterating this set of equations we find a unique completely attractive nontrivial fixed point, which reflects the self-criticality of the stationary state and identifies one of the mechanisms for the generation of SOC systems. In fact, the coupling between the dynamics (energy flux) and the stationarity condition provides a nonlinear feedback mechanism that is responsible for the attractive nature of the RG flow. Moreover, the method allows us to identify the universality class of various models from the basin of attraction of the RG fixed point. In order to evaluate the critical exponents, we directly use the fixed-point stationary and dynamical scale-invariant properties of the systems, in the spirit of the FST method. This allows us to overcome the problems given by the complete attractiveness of the RG flow, i.e., absence of relevant control parameters.

Finally we apply our scheme to the nonconservative sandpile models where a dissipation parameter is considered. The result is that the introduction of dissipation turns the fixed point into a trivial one, and this means that the system is no longer in a critical state. This is in agreement with the simulation on dissipative sandpile models [12], while other dissipative models [13] appear to belong to different universality classes.

The outline of the paper is as follows. In Sec. II we describe the sandpile automata and introduce the scaling laws characterizing the system. In Sec. III we define the parameters that describe the stationary and dynamical properties of the critical state at a generic scale of coarse graining. In Sec. IV we find the renormalization transformation for these parameters and analyze their flow under scale transformation. In Sec. $V$ we evaluate the critical exponents for the avalanche activity from the fixed-point dynamics of the system. In Sec. VI we apply our RG scheme to the class of dissipative sandpile models. In Sec. VII we summarize the results and present conclusions.

\section{SANDPILE MODELS}

In order to visualize the SOC idea, Bak, Tang, and Wiesenfeld proposed a class of simple dynamical systems popularly known as sandpile models [1]. These models are cellular automata defined on a $d$-dimensional lattice where each site $i$ is associated with a variable $E(i)$. This variable is the "energy" stored on each node of the lattice, and can represent several physical quantities such as the height of the pile, mechanical stress, etc. Starting from an arbitrary initial distribution of $E(i)$, one adds an input energy $\delta E$ on a randomly chosen site every time step, such that

$$
E(\mathbf{i}, t+1)=E(\mathbf{i}, t)+\delta E .
$$

When the energy of a site violates a certain stability criterion, a set of rules are applied for determining how the system rearranges itself. These rules are applied until no relaxation occurs, i.e., all the sites are stable. The stability criterion as well as the dynamics of the relaxation processes depend upon the particular automaton. However, it is possible to identify some common characteristics that seem to define the universality classes of the various models. The most studied class is that of nondirected critical height models. In this case, the stability criterion is given by a critical threshold $E_{c}$. When the energy of a site reaches this critical value, a relaxation event takes place and the energy is distributed to neighbors in the following way:

$$
\begin{aligned}
& E(\mathbf{i}) \rightarrow E(\mathbf{i})-\sum_{\mathrm{e}} \Delta E(\mathbf{e}), \\
& E(\mathbf{i}+\mathbf{e}) \rightarrow E(\mathbf{i}+\mathbf{e})+\Delta E(\mathbf{e}),
\end{aligned}
$$

where e denotes the unit vectors. If the energy of some neighbors exceeds the threshold value, they become unstable on their turn, the dynamical process continues, and an avalanche is generated. These models are then called nondirected if, at least on average, they satisfy the condition

$$
\mathrm{J}=\sum_{\mathrm{e}} \Delta E(\mathrm{e}) \mathrm{e}=0,
$$

which means that dynamical rule is isotropic. Usually open boundary conditions are used so that energy can leave from the system, but other conventions are possible. Independent of the initial conditions, the system organizes itself in a critical state characterized by power law distributions and therefore without any characteristic length or time scale. The interesting point is that, unlike ordinary critical phenomena, no fine tuning of any control parameters is necessary to reach this state. In other words, the critical state is an attractor for the dynamics, and the parameters of the system flow spontaneously to the critical value.

The model originally introduced by Bak, Tang, and Wiesenfeld [1] (BTW) is a discrete two-dimensional automaton in which $E_{c}=4$ and $\Delta E=1$. This model has been generalized successively by Zhang and co-workers $[14,15]$, allowing that the energy takes a continuous value and defining $\Delta E=E(i) / 4$. Another interesting model belonging to this class is the two state model of Manna [16]. Here the energy can take only two values $\left(E_{c}=2\right)$, and when a relaxation event occurs the energy is distributed to two randomly chosen nearest-neighbor sites.

In order to characterize the critical state of these systems, a set of critical exponents which refers to the avalanche amplitudes has been defined. The distribution of avalanches is described by a power law behavior

$$
P(s) \sim s^{-\tau},
$$

in which $s$ is the number of sites involved in the relaxation process. Also, the relation that characterizes the dynamics of the problem by linking time and linear extension in a single avalanche follows the scaling law 


$$
t \sim r^{z}
$$

where $z$ is the dynamical critical exponent. Several other quantities which obey scaling laws can be defined for the avalanche. For instance, the duration $t$ and linear size $r$ have a power law distribution characterized by the exponents $\alpha$ and $\lambda$, respectively. Finally, scaling laws mutually connect the quantities $t, s$, and $r$. All previous exponents depend upon the dimensionality of the system, and scaling relations among them can be identified by using general arguments [17]. In $d=2$, where the avalanches are compact, we have

$$
\begin{aligned}
& \lambda=1+2(\tau-1), \\
& \alpha=1+\frac{2(\tau-1)}{z},
\end{aligned}
$$

and it is possible to define $\tau$ and $z$ as the independent critical exponents.

In the past few years extensive numerical simulations have been devoted to the study of discrete and continuous sandpile models, and critical exponents have been measured for various dimensionality in several models [1,14,16-20]. The BTW, Zhang, and two state models are supposed to belong to the same universality class, and the exponents measured are very close, although some discrepancies are observed, probably due to finite size effects. Other classes of models have also been studied. In the critical slope models (CSM) [18] and in the critical Laplacian model (CLM) [18] a relaxation event takes place when the local gradient or local Laplacian, respectively, reaches a critical threshold. These models show critical exponents that are very different with respect to the critical height models, and therefore define different universality classes. The same is obtained by introducing a preferred direction in the avalanche dynamics as well as in the directed sandpile models [19].

Two main theoretical approaches have been followed in the past. The first refers to stochastic nonlinear equations [21,22]. The main difficulty of this kind of approach is that avalanches are not clearly defined and some information about the system is lost. The second type of theoretical approach involves the group theory developed by Dhar [23], who studied a class of Abelian sandpile models by introducing a general formalism to characterize the critical state of these models. However, until now only some simple models, such as the directed model and the BTW on the Bethe lattice, have been solved exactly following this approach. Finally, a field theory approach for the SOC properties of a model related to invasion percolation was recently proposed [24].

In what follows, we will show that a real space renormalization group approach recently developed [11] allows us to calculate analytically the critical exponents $\tau$ and $z$ and to clarify the SOC nature of the sandpile models.

\section{DYNAMICAL AND STATIONARY PARAMETERS IN SANDPILE MODELS}

Our discussion will refer to the critical height models. As was previously shown, these models are cellular auto- mata defined on a lattice. A variable (energy) is assigned to each site; energy is then added randomly to the system. When the energy of a site reaches a critical value, its energy is released to the neighboring sites in various ways. These may become unstable in their turn, and so on. The system usually has open boundary conditions which allow the energy to dissipate outside. Given this picture of the system we can, in full generality, characterize three classes of sites (Fig. 1).

(i) Stable sites are those whose energy is far from the threshold value. This implies that the addition of a "quantum" of energy will not induce relaxation.

(ii) Critical sites are those whose energy is critical in the sense that the addition of a quantum of energy will induce relaxation.

(iii) Unstable sites are those that will relax at next time step according to the specific rules assigned.

An example of this relaxation is shown in the lower part of Fig. 1. In this case the relaxation of the central site induces a redistribution of the energy over two of the neighbors, as in the model of Manna [16], and these become critical in their turn. The stationary critical state of the system will be described by the probability $\rho$, which defines the density of critical sites. This parameter characterizes the equilibrium in the energy balance and can be viewed as the control parameter of the model. However, it is not a relevant control parameter, as for example the temperature in the Ising model, and it automatically reaches its critical value during the dynamical evolution of the system.

We can now extend the characterization of the stationary properties at a generic scale $b$, by considering coarse grained variables (Fig. 2). Independent of the minimal scale, a coarse grained cell of size $b$ is stable if the addition of a quantum of energy $\delta E(b)$, corresponding to scale $b$, does not induce relaxation into neighboring cells. Of course internal relaxations can occur, but as long as they do not affect other cells we consider the cell stable. Conversely, a cell is critical if the addition of $\delta E(b)$ induces relaxation into some neighboring cell(s). From these definitions it is then possible to introduce the pa-

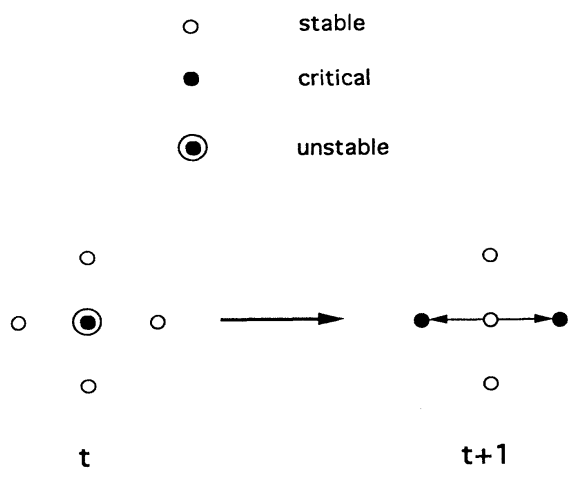

FIG. 1. Upper part: classification of the three possible classes of sites. Stable (white) sites do not relax if a quantum of energy is added. Critical (black) sites would relax if energy were added. Unstable (encircled) sites will relax at the next time step. In the lower part we show an example of relaxation dynamics. 

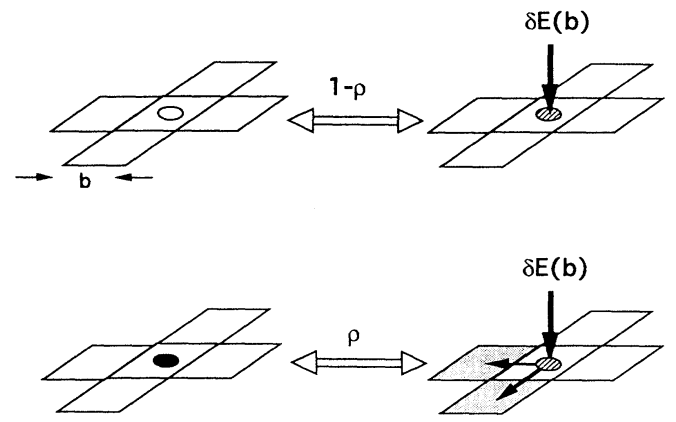

FIG. 2. Characterization of the static properties of coarse grained cells. A cell of generic scale $b$ is stable (white) if $\delta E(b)$ does not induce relaxation in the neighboring cells of the same size. In the opposite case the cell is critical (black).

rameter $\rho(b)$, that gives the density of critical cells at a generic scale $b$ of coarse graining.

We can now follow the same idea to characterize the dynamical properties of the system at a generic scale $b$. A cell of size $b$ relaxes if subrelaxation processes span the cell and transfer energy to some neighboring cell. Independent of the minimal scale dynamics such a relaxation process, for a coarse grained cell of size $b$ in a square lattice, can lead to four possible situations as shown in Fig. 3. Energy can be transferred to one, two, three, or four neighbors with a probability distribution defined by the vector

$$
\vec{P}=\left(p_{1}, p_{2}, p_{3}, p_{4}\right),
$$

Here the probabilities $p_{i}$ refer to the sum of all the processes that affect the corresponding number of neighboring cells, independent of their positions, and satisfy the normalization condition $\Sigma p_{i}=1$. The vector $\vec{P}(b)$ then

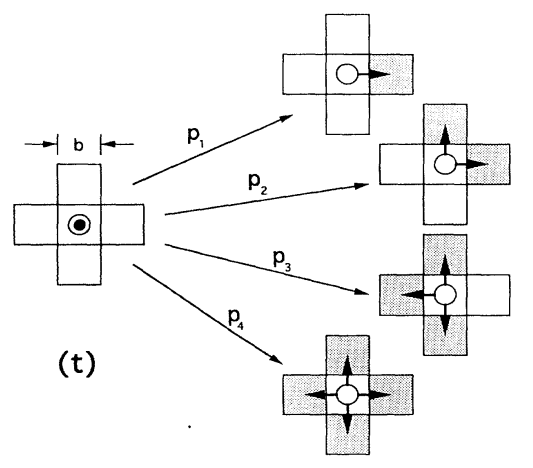

$(t+1)$

FIG. 3. No matter what the small scale dynamics, at a generic scale $(b)$ relaxation can occur in the four possible ways shown. These four possibilities characterize the phase space of the dynamics of the system that will be used in the relaxation scheme. The probabilities refer to the number of neighboring cells influenced by the relaxation, independent of their position. characterizes the phase space for the relaxation dynamics at a generic scale of coarse graining. In this framework we can define the vector that describes the small scale dynamics of the various models. For instance, the model of Manna [16] distributes energy to only two sites, and therefore the small scale dynamics is given by the vector $\vec{P}^{(0)} \equiv(0,1,0,0)$. The BTW model [1] distributes energy to all four neighbors, and corresponds to $\vec{P}^{(0)} \equiv(0,0,0,1)$. We are going to see that, under scale transformation and renormalization, these two vectors evolve to the same fixed point, so that the two models belong to the same universality class. By using this description we show that both the stationary and dynamical properties of sandpile models are then fully characterized by the distribution $(\rho(b) ; \vec{P}(b))$, which is therefore the natural candidate for a renormalization procedure.

\section{RENORMALIZATION EQUATIONS}

We now proceed to define a renormalization procedure for the relaxation dynamics. We will use a cell-to-site transformation on the square lattice, in which each cell at scale $b$ is formed by four subcells at scale $b / 2$. Every cell at the larger scale is then characterized by the index $\alpha$, ranging from one to four (Fig. 4), that gives the number of critical subcells. Therefore, in the stationary state the weight of each configuration $\alpha$ is given by the probability of having a corresponding number of critical subcells

$$
W_{\alpha}(\rho)=n_{\alpha} \rho^{\alpha}(1-\rho)^{4-\alpha},
$$

where $n_{\alpha}$ is a normalization factor. Here we consider the energy distribution of the stationary state as uncorrelated [20]. In fact, correlations among sites are developed only during the dynamical evolution of the avalanche, and average out in the stationary state. Consequently, in our scheme correlations are taken into account in the renormalization equations for the dynamics. In order to define the renormalization transformation, we assume that one of the critical subcells at scale $b / 2$ relaxes, and study how energy is distributed to the neighboring cells of size

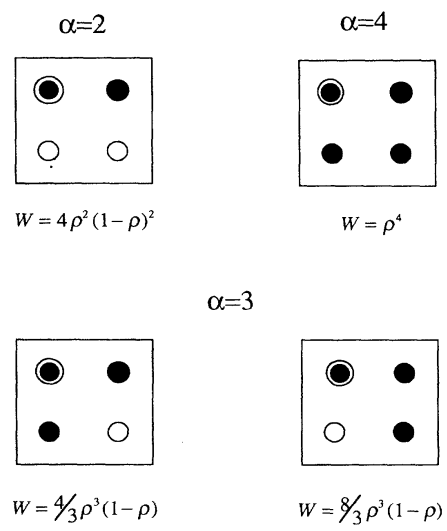

FIG. 4. A $2 \times 2$ cell is characterized by the number $\alpha$ of critical sites. We show the four possible starting configurations, with their relative weight, involved in the renormalization of the dynamical parameters. 
$b$. Here we consider a transformation defined by the following rules.

(i) Each critical subcell relaxes if it receives energy from one or more neighboring subcells. Conversely, relaxation processes cannot occur in a stable subcell.

(ii) Every series of relaxation processes $p_{n^{\prime}}^{(k)}$, where the index $(k)$ refers to the scale $b / 2$, that span the starting cell and transfer energy to $n$ neighboring cells is renormalized in the correspondent process $p_{n}^{(k+1)}$, where $(k+1)$ refers to the scale $b$.

(iii) We consider only those connected series of processes that span the cell from left to right or top to bottom. This spanning rule implies that only processes extending over the size of resulting length scale contribute to the renormalized dynamics. Moreover it ensures the connectivity properties of the avalanche in the renormalization procedure.

An example of such a process is shown in Fig. 5. In this case $\alpha=2$, and the process shown refers to the probability that the unstable subcell relaxes toward the other critical subcell [Fig. 5(b)]. This occurs with probability $(1 / 4) p_{1}^{(k)}$. At this point we consider the probability that the next relaxation event at scale $b / 2$ involves two neighboring sites of size $b / 2$, one inside and one outside the original cell of size $b$ [Fig. 5(c)]. This occurs with probability $(2 / 3) p_{2}^{(k)}$. This series of relaxation processes at scale $b / 2$ contributes to the probability $p_{1}^{(k+1)}$ that characterizes the relaxation processes at scale $b$. By summing over all the processes that lead to $p_{1}^{(k+1)}$ one obtains, for $\alpha=2$,

$$
\begin{aligned}
p_{1}^{(k+1)}= & \left(\frac{1}{4} p_{1}^{(k)}+\frac{1}{6} p_{2}^{(k)}\right)\left(\frac{1}{2} p_{1}^{(k)}+\frac{2}{3} p_{2}^{(k)} \frac{1}{2} p_{3}^{(k)}\right) \\
& +\left(\frac{1}{6} p_{2}^{(k)}+\frac{1}{4} p_{3}^{(k)}\right)\left(\frac{1}{2} p_{1}^{(k)}+\frac{1}{6} p_{2}^{(k)}\right) \\
& +\left(\frac{1}{6} p_{2}^{(k)}+\frac{1}{4} p_{3}^{(k)}\right)\left(\frac{3}{4} p_{1}^{(k)}+\frac{1}{2} p_{2}^{(k)} \frac{1}{4} p_{3}^{(k)}\right) .
\end{aligned}
$$

In a similar way one can also write the expression for $p_{2}^{(k+1)}, p_{3}^{(k+1)}$, and $p_{4}^{(k+1)}$. In addition, these probabilities are normalized for each configuration $\alpha$.

In a general way, for each starting cell, we can define the set $\omega_{n}$ of process series at scale $b / 2$ that renormalize in a $p_{n}^{(k+1)}$ process at scale $b$ and the respective statistical weight $f_{\omega_{n}}$ of this set, which is a function of parameters $p_{n^{\prime}}^{(k)}$ at scale $b / 2$. As an example, with this definition we have that $f_{\omega_{1}}$ for the starting cell $\alpha=2$ is given by the term on the right-hand side of Eq. (10). For each starting

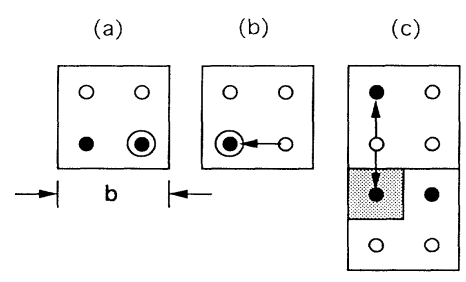

Microscopic relaxation processes (d)

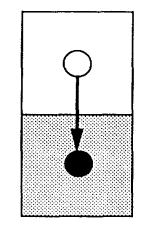

Coarse grained relaxation
FIG. 5. Example of the renormalization scheme for the relaxation dynamics. For details, see the text. cell we can therefore write the renormalization equations as

$p_{n}^{(k+1)}(\alpha)=\sum_{\left\{\omega_{n}\right\}_{\alpha}} f_{\omega_{n}}\left(\left\{p_{n^{\prime}}^{(k)}\right\}\right), \quad n, n^{\prime}=1, \ldots, 4$,

where the statistical weight is normalized so that

$$
\sum_{n} \sum_{\omega_{n}} f_{\omega_{n}}=1
$$

Finally we have to average this equation over all possible starting configurations $\alpha$, obtaining the following complete renormalization equations:

$$
\begin{aligned}
p_{n}^{(k+1)} & =\sum_{\alpha=2}^{4} W_{\alpha}\left(\rho^{(k)}\right) p_{n}^{(k+1)}(\alpha) \\
& =\sum_{\alpha=2}^{4} W_{\alpha}\left(\rho^{(k)}\right) \sum_{\left\{\omega_{n}\right\}_{\alpha}} f_{\omega_{n}}\left(\left\{p_{n^{\prime}}^{(k)}\right\}\right) .
\end{aligned}
$$

Here the configuration $\alpha=1$ is not included in the renormalization of the dynamics, because it cannot lead to relaxation processes that span the whole cell of size $b$. The explicit writing of the above equations is extremely complex, and the detailed calculation is reported in the Appendix.

The renormalization of the relaxation processes alone would lead to trivial fixed points because the system simply decays. In order to describe the dynamics of the stationary critical state it is necessary to couple the dynamics to a stationarity condition similar to the one used in Ref. [15] to define the average energy of the critical state. The stationary state is characterized by the balance between the energy that goes in and the energy that goes out of the system. This means that, given a cell of size $b$, on average, the energy that goes into the cell has to be equal to the energy that goes out. If we inject a quantum of energy $\delta E(b)$, the probability that the cell relaxes is $\rho^{(k+1)}$ and, once this happens, it can occur with the four possible situations described previously (Fig. 3). This leads to the equilibrium condition

$$
\begin{aligned}
\delta E(b)=\rho^{(k+1)}[ & \delta E(b) p_{1}^{(k+1)}+2 \delta E(b) p_{2}^{(k+1)} \\
& \left.+3 \delta E(b) p_{3}^{(k+1)}+4 \delta E(b) p_{4}^{(k+1)}\right] .
\end{aligned}
$$

that then provides the renormalized density of critical sites at scale $b$, independently of the definition of $\delta E(b)$. Note that we have considered transferred energy proportional to the number of neighboring sites involved in the relaxation process. This has to be on average for isotropic relaxation, i.e., the energy is proportional to the avalanche front, and can be tested self-consistently with our method.

The complete structure of our renormalization group is therefore the following:

$$
\begin{aligned}
& p_{n}^{(k+1)}=\sum_{\alpha=2}^{4} W_{\alpha}\left(\rho^{(k)}\right) \sum_{\left\{\omega_{n}\right\}_{\alpha}} f_{\omega_{n}}\left(\left\{p_{n^{\prime}}^{(k)}\right\}\right), \\
& \rho^{(k+1)}=\left(p_{1}^{(k+1)}+2 p_{2}^{(k+1)}+3 p_{3}^{(k+1)}+4 p_{4}^{(k+1)}\right)^{-1} .
\end{aligned}
$$

In this scheme the equilibrium condition couples the dynamical properties to the stationary ones, and provides a feedback mechanism that is an essential element for the 
process of self-organization [6] and for the definition of the nonlocal properties of the dynamics. The approximations involved in our approach are those usually present in real space renormalization group (RSRG) methods $[25,26]$ due to the specific implementation of the spanning condition and to closure of the renormalization equations. In fact, proliferation effects due to multiple relaxations and sites becoming critical during the dynamical process are not considered in our scheme. However, these effects are less and less important on greater scale [20], and can be considered asymptotically irrelevant.

Given this scheme, the flow diagram and the relative fixed points in the phase space of the parameters $(\rho, \vec{P})$ can be studied. In order to do this, we start from a small scale state characterized by $\left(\rho^{(0)} ; \vec{P}^{(0)}\right)$ and study how it will evolve under scale transformation. If we start with the small scale dynamics of the two state model [16] we have $\overrightarrow{\boldsymbol{P}}^{(0)}=(0,1,0,0)$. No matter what the value of the density of critical sites, the system $\left(\rho^{(0)} \neq 0\right)$ flows in the same nontrivial fixed point. The same happens if we start from the small scale dynamics of the BTW model $\left[\overrightarrow{\boldsymbol{P}}^{(0)}=(0,0,0,1)\right]$. In Table I we show the evolution under scale transformation for both models starting from different values of the density of critical sites. Both models lead to the same asymptotic $(k \rightarrow \infty)$ fixed-point dynamics $\left(\rho^{*} ; \vec{P}^{*}\right)$, so they belong to the same universality class. In addition, we have checked that all models whose stationary and dynamical properties can be parametrized in such a way also belong to this universality class. In fact, the fixed point is attractive in the whole phase space, so that the parameters $(\rho ; \vec{P})$ evolve spontaneously toward their critical value. Therefore, in this perspective we are able to understand the self-organized nature of the critical stationary state of sandpile models.

It is worth remarking that the nonlinear coupling between the parameter $\rho$ and the system's dynamics $\vec{P}$ generates a feedback mechanism among the stationary and

TABLE I. Renormalization transformation for the stationary $(\rho)$ and dynamical $\left(p_{n}\right)$ properties for two sandpile models. The index $k$ refers to a change of scale. The limit $k \rightarrow \infty$ identifies the attractive fixed point $\left(\rho^{*} ; \vec{P}^{*}\right)$ that is the same for both models.

\begin{tabular}{|c|c|c|c|c|c|}
\hline$k$ & $\rho$ & $p_{1}$ & $p_{2}$ & $p_{3}$ & $p_{4}$ \\
\hline \multicolumn{6}{|c|}{ Manna two-state model } \\
\hline 0 & 0.1 & 0 & 1 & 0 & 0 \\
\hline 1 & 0.612 & 0.436 & 0.495 & 0.068 & 0.001 \\
\hline 2 & 0.575 & 0.405 & 0.463 & 0.118 & 0.013 \\
\hline 3 & 0.542 & 0.362 & 0.456 & 0.158 & 0.024 \\
\hline 4 & 0.518 & 0.324 & 0.434 & 0.188 & 0.033 \\
\hline$\infty$ & 0.468 & 0.240 & 0.442 & 0.261 & 0.057 \\
\hline \multicolumn{6}{|c|}{ BTW four-state model } \\
\hline 0 & 0.9 & 0 & 0 & 0 & 1 \\
\hline 1 & 0.252 & 0 & 0 & 0.033 & 0.967 \\
\hline 2 & 0.308 & 0 & 0.012 & 0.726 & 0.262 \\
\hline 3 & 0.353 & 0.030 & 0.261 & 0.553 & 0.152 \\
\hline 4 & 0.388 & 0.090 & 0.357 & 0.437 & 0.116 \\
\hline$\infty$ & 0.468 & 0.240 & 0.442 & 0.261 & 0.057 \\
\hline
\end{tabular}

dynamical properties of the models. This mechanism is fundamental in order to have a nontrivial fixed point. Recently various authors $[6,8]$ pointed out that a similar mechanism could be a general way to produce selforganized criticality in extended open systems. This concept relies on the idea of a feedback of the order parameter onto the control parameter. For instance, let us consider the continuous model for sandpiles [27]. In this case the order parameter is the flux $J$ of sand grains that flow out of the system, and the control parameter is the sandpile's slope $\theta$. As usual the behavior of the order parameter is $J \sim\left(\theta-\theta_{c}\right)^{\beta}$ for $\theta>\theta_{c}$, and $J=0$ per $\theta<\theta_{c}$, where $\theta_{c}$ is the critical slope. However, an initial slope larger than the critical value generates large avalanches which bring the slope back to its critical value. The contrary will happen if the slope is lower than the critical value. This picture is a clear example of the nonlinear feedback of the order parameter on the control parameter in order to obtain the attractiveness of the critical slope. In our scheme, it is easy to note that $\vec{P}$ and $\rho$ correspond to the flux $J$ and the control parameter $\theta$, respectively, so that we can show in a mathematical way the relevance of the feedback mechanism.

\section{CALCULATION OF THE CRITICAL EXPONENTS}

From the previous discussion we have shown that the fixed-point parameters $\left(\rho^{*} ; \vec{P}^{*}\right)$ provide a complete description of the critical state. However, the complete attractiveness of the fixed point corresponds to a lack of relevant control parameters in the model, and the critical exponents cannot be found using the usual method. In fact, in usual critical phenomena, the exponents are derived from the derivative of the $R G$ transformation with respect to the critical parameter. In SOC models this is impossible because the fixed point is attractive, and the exponents that are related to the distance from the critical point do not exist. For this reason we use a method analogous to the FST approach to fractal growth [9] which is related directly to the fixed point parameters.

The avalanche exponent $\tau$ can be obtained as follows. The nontrivial fixed point with respect to the scale transformation corresponds to diverging characteristic lengths for the system [26]. Because these lengths set the natural scale for the system, their divergence at the critical points signals the loss of scale, and this guarantees that the properties of the system will be described by homogeneous functions of the physical parameters. Therefore we can assume a power law avalanche distribution and relate the exponent $\tau$ to the fixed-point properties $\left(\rho^{*} ; \vec{P}^{*}\right)$ shown in Table I. The first step is to transform the avalanche distribution into a distribution for the size $r$ of the clusters. In $d=2$, it has been shown analytically [28] and from simulations [20] that the avalanche clusters are compact, and using the relation $s \sim r^{2}$ we obtain

$$
P(r) d r \sim P(s) d s \sim r^{(1-2 \tau)} d r .
$$

By using the discrete length scales $b^{(k)}=2^{(k)} b_{0}$ of our RG scheme, we define the parameter $K$ as the relative probability that an active relaxation process is limited between the scales $b^{(k)}$ and $b^{(k+1)}$ and does not extend further. 
This can be expressed as

$$
K=\frac{\int_{b^{(k)}}^{b^{(k+1)}} P(r) d r}{\int_{b^{(k)}}^{\infty} P(r) d r}=1-2^{2(1-\tau)} .
$$

The parameter $K$ is defined by the conditions that a relaxation event occurs on a scale $b^{(k+1)}$ with the dynamics defined by $p_{n}^{(k+1)}(n=1,4)$, and that this process stops and does not affect the neighboring cells. Asymptotically $(k \rightarrow \infty)$, we can therefore express $K$ in terms of our fixed-point parameters in the following way:

$K=p_{1}^{*}\left(1-\rho^{*}\right)+p_{2}^{*}\left(1-\rho^{*}\right)^{2}+p_{3}^{*}\left(1-\rho^{*}\right)^{3}+p_{4}^{*}\left(1-\rho^{*}\right)^{4}$.

This equation gives the probability that a generic relaxation process affects only stable sites and therefore does not propagate on length scales larger than the scale of the starting cell. Note that this expression is different from the quantity $\left(1-\rho^{(k+1)}\right)$ that includes the situation in which no relaxation events occur even at scale $b^{(k)}$. By inserting into the previous equation the values of the fixed-point parameters and using Eq. (17), the exponent $\tau$ is given by

$$
\tau=1-\frac{1}{2} \frac{\log (1-K)}{\log (2)}=1.253 .
$$

This value of $\tau$ is in good agreement with large computer simulations on the BTW and two state model which give $\tau=1.22[18]$ and 1.28 [16], respectively.

The other independent critical exponent is the dynamical exponent $z$. In this case the calculation results are rather laborious because we also need the knowledge of the whole form of the renormalized dynamics. From the scaling laws at the fixed point we have that the average time of a dynamical process scales with the length size as

$$
t_{k} \sim\left(b^{(k)}\right)^{z} .
$$

Using the discretized length of our scheme, the time scale of a relaxation event at scale $b^{(k+1)}$ and one at scale $b^{(k)}$ are related, in the asymptotic limit $(k \rightarrow \infty)$, by the relation

$$
\frac{t_{k+1}}{t_{k}}=(2)^{z}
$$

The time scale $t_{k+1}$ can be obtained as a function of the time scale $t_{k}$ from the RG equations. In fact, the renormalized dynamical process is given by the weighted average of the series of subprocesses at scale $b^{(k)}$, whose time scale is $t_{k}$. Therefore we have

$$
t_{k+1}=\langle t\rangle t_{k}
$$

where $\langle t\rangle$ is the average number of subprocesses at scale $b^{(k)}$ needed to have a relaxation process at scale $b^{(k+1)}$, and it represents the time scaling factor. By using our RG scheme, the above average can be written as

$$
\langle t\rangle=\sum_{\alpha, n} W_{\alpha}\left(\rho^{(k)}\right) \sum_{\left\{\omega_{n}\right\}_{\alpha}} f_{\omega_{n}}\left(\left\{p_{n^{\prime}}^{(k)}\right\}\right) t_{\omega_{n}},
$$

having considered that $t_{\omega_{n}}$ is the number of noncontemporary relaxation events in each series of subprocesses $\omega_{n}$. By inserting the fixed-point parameters into the calculation of $\langle t\rangle$, from Eq. (21) we obtain the following result for the dynamical exponent

$$
z=\frac{\log \langle t\rangle}{\log 2}=1.168 \text {. }
$$

This value can be readily compared to existing estimates of $z$, and our analytical prediction is in very good agreement with the numerical results that give $z \simeq 1.20$ $[16,18]$. The value of the dynamical exponent we reported in Ref. [11] was obtained with a preliminary simplified scheme of calculation, and is slightly different from that obtained with the present complete calculation. We want to emphasize that in this case we used not only the fixedpoint parameters, as in the calculation of $\tau$, but also the complete form of the scale invariant dynamical evolution from scale $b / 2$ to scale $b$. For this reason the calculation of the dynamical exponent can be improved by using greater cells to obtain the renormalization transformation.

The other critical exponents of the system can be derived from the scaling relations (6) and (7) using the values obtained for $\tau$ and $z$. In Table II the values of the critical exponents calculated with our RG scheme are compared with the best estimates from numerical simulations of the BTW model, and it can be noted that the agreement is very good. These results are obtained using the simplest renormalization scheme, and it is also possible to consider more complicated calculation schemes (larger cells, spanning condition, etc.) in order to improve the numerical values.

With our RG scheme we therefore provide a complete characterization of the critical state, and obtain analytically the full set of the exponents defining the critical behavior of sandpile models. Furthermore, this approach contains some novelties that make it substantially different from usual RSRG calculations and particularly suitable in all SOC problems. In fact, our discussion referred to the two-dimensional case; however, it is conceptually straightforward to extend the method to the case $d>2$ and to variations of the original model. Also the universality of sandpile models with respect to different Bravais lattices can be addressed with our model. The local characteristics of the lattice, such as the number of nearest neighbors, determine the form of the dynamical

TABLE II. In this table the values of the critical exponents calculated with our RG scheme are summarized. For comparison we also list the values obtained from large scale simulations on the BTW model $(d=2)[18]$.

\begin{tabular}{lllll}
\hline \hline & \multicolumn{1}{c}{$\tau$} & \multicolumn{1}{c}{$\alpha$} & \multicolumn{1}{c}{$\lambda$} & \multicolumn{1}{c}{$z$} \\
\hline RG scheme & 1.253 & 1.432 & 1.506 & 1.168 \\
BTW simulations & 1.22 & 1.38 & 1.44 & 1.21 \\
\hline \hline
\end{tabular}


vector and the critical fixed-point value of the parameters, but the critical exponents should be universal and determined only by the large scale properties of the system. Thus, by using our method for sandpile models defined on triangular lattice, we expect to find a completely different fixed-point dynamics (actually even the phase space of the dynamics is different), but critical exponents very close to those obtained for the square lattice. Preliminary calculation using this approach on the triangular lattice gives an exponent $\tau$ that is in very good agreement with those obtained here, although the critical parameters are very different [29].

\section{RENORMALIZATION SCHEME FOR THE NONCONSERVATIVE CASE}

Nonconservative sandpile automata have been introduced to consider a relaxation dynamics where part of the involved energy is dissipated. Denoting by $\Delta E$ and $\Delta E_{\text {out }}$, respectively, the energy lost by the relaxing site and the energy really transferred to the nearest neighbors, we can identify the fraction of energy that disappears from the system as

$$
\gamma=1-\frac{\Delta E_{\text {out }}}{\Delta E}
$$

The dissipation parameter $\gamma$ quantifies the level of nonconservation of the system. We can have $\gamma \neq 0$ for every relaxation event (local dissipation) but $\langle\gamma\rangle=0$ (global conservation) averaging over many processes. If the dissipation is global $(\langle\gamma\rangle \neq 0)$ the SOC behavior is destroyed and a characteristic length is introduced, as was shown by computer simulations [12]. This means that the avalanche distribution follows an exponential behavior and that ${ }^{-}$the characteristic length for the avalanche size $l_{c}$ diverges, as $\gamma \rightarrow 0$, such that

$$
l_{c} \sim|\gamma|^{-v},
$$

in complete analogy with the usual critical phenomena.

In order to apply our renormalization scheme in practice, we use the following definition of a nonconservative sandpile with global dissipation. The nonconservation is introduced by the probability $\gamma$ that a relaxation process dissipates its entire energy. Instead, with probability $(1-\gamma)$, the relaxation event transfers the energy following the usual dynamical rules. This model is therefore a particular case of the global dissipative class introduced in [12], where the probability $\gamma$ is the dissipation parameter. Trivially, for $\gamma=0$ the model becomes conservative, while in the limit $\gamma \rightarrow 1$ it is completely dissipative (no relaxation occurs).

We can follow the scheme used in the previous sections considering that the parameter $\gamma$ should also be renormalized. Though the renormalization of the dynamics can be performed in the same way as in the conservative case, we have to consider explicitly the effect of the dissipation parameter. This modifies Eq. (13) into

$$
\begin{array}{r}
p_{n}^{(k+1)}=\sum_{\alpha} W_{\alpha}\left(\rho^{(k)}\right) \sum_{\left\{\omega_{n}\right\}_{\alpha}} f_{\omega_{n}}\left(\left\{p_{n^{\prime}}^{(k)}\right\}, \gamma^{(k)}\right), \\
n, n^{\prime}=1, \ldots, 4,
\end{array}
$$

where $\gamma^{(k)}$ denotes the dissipation parameter at scale $b^{(k)}$. The scheme used to evaluate explicitly the weight $f_{\omega_{n}}$ is modified slightly because we must take into account the possibility that one or more relaxation processes dissipate their energy. Thus, as previously, only the critical sites can induce a relaxation process, and the spanning condition is the same as for conservative sandpiles. However, there are two possible cases for every relaxation process. With probability $(1-\gamma)$ the relaxation process transfers its energy to the neighboring sites. Conversely, with probability $\gamma$ the process dissipates its energy and does not affect other sites. Thus the weight of every series of subprocesses will have an additional factor $\gamma^{q}(1-\gamma)^{s}$, where $q$ and $s$ are the number of subprocesses dissipating and transferring energy, respectively. In Fig. 6 is shown a renormalization example in which some subprocesses are dissipative.

It is clear that a site has to be considered critical independently if it dissipates or transfers energy during the relaxation event. Therefore, the stationarity condition given by the energy balance now reads as

$$
\begin{aligned}
\delta E= & \left(1-\gamma^{(k+1)}\right) \rho^{(k+1)} \delta E \sum_{n=1}^{4} n p_{n}^{(k+1)} \\
& +\gamma^{(k+1)} \rho^{(k+1)} \delta E \sum_{n=1}^{4} n p_{n}^{(k+1)}
\end{aligned}
$$

where the first term represents the average energy transferred to neighbors, while the second refers to the average dissipated energy. It then follows, by summing the two terms of the above equation, that the stationarity condition is the same as in the conservative case.

Next we study the renormalization of the dissipation parameter. In order to do this we can consider the renormalized dissipation $\gamma^{(k+1)}$ as the ratio between the energy dissipated and the total energy involved in the relaxations at scale $b^{(k+1)}$ :

$$
\gamma^{(k+1)}=\frac{\Delta E_{\mathrm{diss}}^{(k+1)}}{\Delta E_{\mathrm{out}}^{(k+1)}+\Delta E_{\mathrm{diss}}^{(k+1)}} .
$$

Here $\Delta E_{\text {diss }}^{(k+1)}$ and $\Delta E_{\text {out }}^{(k+1)}$ represent the energy dissipated and the energy transferred, respectively, by the coarse grained cell, conditionally to the occurrence of a relaxation process. By analyzing the various subprocesses in-

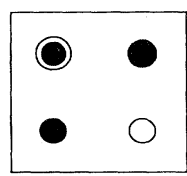

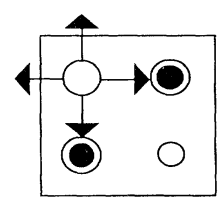

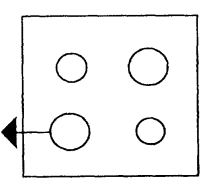

FIG. 6. Renormalization example in the dissipative case. (a) Starting cell. (b) The unstable site relaxes with a process affecting four neighbors. The two critical subcells become unstable. (c) Only one of the two subcells relaxes, transferring energy to one neighbor site. The second subcell dissipates its energy. The weight of these processes is $f=\gamma(1-\gamma)^{2} p_{4} p_{1}$. 
side the cell, the explicit renormalization equation can be written expressing the quantities of the right term as a function of the parameters at scale $b^{(k)}$ :

$$
\begin{gathered}
\Delta E_{\text {out }}^{(k+1)}=\sum_{\alpha, n} W_{\alpha}\left(\rho^{(k)}\right) \sum_{\left\{\omega_{n}\right\}_{\alpha}} f_{\omega_{n}}\left(\left\{p_{n^{\prime}}^{(k)}\right\}, \gamma^{(k)}\right) E_{\text {out }}\left(\omega_{n}\right) \\
\begin{array}{c}
\Delta E_{\text {diss }}^{(k+1)}=\sum_{\alpha, n} W_{\alpha}\left(\rho^{(k)}\right) \sum_{\left\{\omega_{n}\right\}_{\alpha}} f_{\omega_{n}}\left(\left\{p_{n^{\prime}}^{(k)}\right\}, \gamma^{(k)}\right) E_{\text {diss }}\left(\omega_{n}\right) \\
+G\left(\left\{p_{n^{\prime}}^{(k)}\right\}, \gamma^{(k)}\right) .
\end{array}
\end{gathered}
$$

In the above equations we denote $E_{\text {out }}\left(\omega_{n}\right)$ and $E_{\text {diss }}\left(\omega_{n}\right)$ as the average number of quantums of energy at scale $b^{(k)}$ transferred and dissipated, respectively, during the process $\omega_{n}$. In this calculation, $\delta E\left(b^{(k)}\right) \sum_{n=1}^{4} n p_{n}^{(k)}$ is the average energy that each subprocess can dissipate, namely the average energy it can transfer to the neighboring sites during the usual relaxation. It should be noted that the last term of Eq. (31) takes into account all the processes that dissipate energy without affecting the neighboring cells, so that they are not included in the series $\omega_{n}$. Furthermore by Eq. (29) it is also clear that the renormalization of the dissipation parameter is independent by the definition of $\delta E\left(b^{(k)}\right)$. In Fig. 6 is shown a process that contributes to the renormalization of $p_{2}$ with $E_{\text {out }}(\omega)=3 \delta E\left(b^{(k)}\right)$ and $E_{\text {diss }}(\omega)=\delta E\left(b^{(k)}\right) \sum_{n=1}^{4} n p_{n}^{(k)}$. The last sum takes into account the average energy that the subcells can dissipate.

The calculation is rather laborious but straightforward, and we can finally iterate the obtained renormalization equations. Trivially, starting from the conservative case $\gamma=0$ we obtain the results derived in the previous sections. Conversely, if we start with $\gamma \neq 0$ the flow diagram suffers a dramatic change. The attractive fixed point becomes the trivial one $\gamma^{*}=1$. This implies that at large scale the entire energy added to the system is dissipated, and that the system has a trivial behavior in which no relaxation events occur. Therefore, the presence of a dissipation parameter in the model introduces an upper characteristic length for the avalanches. In this case $\gamma=0$ is an unstable (repulsive) fixed point and we can look at the system in the usual perspective of critical phenomena by considering the dissipation parameter as the relevant control parameter of the model with a critical value $\gamma_{c}=0$. Since this fixed point is repulsive, we can evaluate the exponent linking the dissipation to the avalanche characteristic length [Eq. (26)] with the usual linearization around the unstable fixed point of the RG transformation. By simple general consideration it is possible to see that

$$
\left[\frac{\partial \gamma^{(k+1)}}{\partial p_{i}^{(k)}}\right]_{\gamma=0}=\left[\frac{\partial \gamma^{(k+1)}}{\partial \rho^{(k)}}\right]_{\gamma=0}=0 .
$$

In fact, the renormalization equation for the dissipation parameter [Eq. (29)] is a power series of $\gamma$ itself. This is because $\Delta E_{(\text {diss })}^{(k+1)}$ contains only terms with at least one dissipation event and then with weighting factors that are powers of $\gamma$. Each derivative of Eq. (29) with respect to a parameter other than $\gamma$ is therefore equal to zero in the limit $\gamma \rightarrow 0$. From this it follows that the positive eigenvalue of the linearized RG transformation is

$$
\lambda=\left[\frac{\partial \gamma^{(k+1)}}{\partial \gamma^{(k)}}\right]_{\gamma=0},
$$

and the exponent governing the scaling of the characteristic length is

$$
v=\frac{\log 2}{\log \lambda}=0.67 \text {. }
$$

This value can be compared with those obtained from simulations $v \simeq 0.5$ [12]. This calculation is a vivid illustration of the difference in the calculus of the critical exponent in the SOC case and in the ordinary critical case. The main result is that dissipation introduces a length scale into the system and destroys the SOC properties. This is in agreement with the simulations on systems of the type described here [12]. However, this is not a general result, and a different definition of the nonconservative mechanism can belong to a different universality class. This appears to be the case for the models described in Ref. [13]. The above analysis also shows that our scheme can easily be extended to treat variations of the original sandpile models.

\section{CONCLUSIONS}

In this paper we have presented an alternative renormalization approach suited to a study the SOC state of sandpile models. The method shown in this paper is based on two steps. The first is a renormalization scheme to identify the scale-invariant dynamics of the models. In order to do this we define the proper phase space of parameters in which the $R G$ transformation is constructed, and then introduce the coupling of the renormalization equations to the stationarity condition of the system. This coupling provides the feedback mechanism responsible for the attractiveness of the fixed point. Second, we evaluate the critical exponents of the systems directly from the scale-invariant dynamics instead of the derivative of the RG equations with respect to the relevant critical parameter, that in SOC systems does not exist. These two steps cast the relevant physical elements of the SOC problems in the proper mathematical framework that allows us to clarify the self-critical nature of the SOC problems and to draw a picture of the various universality classes of sandpile models. Moreover, it is important to note that the present general discussion is not affected by the approximations involved in our scheme, even though a more refined treatment of the calculation schemes (larger cells, spanning condition, etc.) can lead to an improvement of the values obtained for the critical exponents.

We have shown in detail the calculation of the renormalization equations in the case of the critical height sandpile models in $d=2$. We analyzed the flow diagram in the phase space of the parameters, and the attractive fixed point was identified. By using fixed-point parameters we are able to calculate analytically the independent critical exponents, namely the avalanche and the dynamical exponent. The values obtained for the critical ex- 
ponents are in very good agreement with the previous estimates from numerical simulations (see Table II).

We also apply our scheme to the case of dissipative sandpile models, finding that the introduction of the dissipation parameter turns the fixed point into a trivial one by introducing a length scale into the systems. This is in agreement with simulations on these models [12], but does not close the issue with respect to other dissipative models such as those of Ref. [13], which probably belong to different universality classes.

The method we have shown can be naturally extended to higher dimensionality $(d>2)$ and to other problems with nonequilibrium stationary states, as for example the Forest Fire model [30].

\section{ACKNOWLEDGMENTS}

It is a pleasure to thank P. Bak, D. Dhar, H. Kaufman, and A. Stella for interesting discussions.

\section{APPENDIX}

The renormalization equations (13) can be obtained explicitly, but the calculation is rather long. In practice we have to enumerate all the processes that span a given cell. Then we assign to each process a statistical weight in terms of $\rho$ and $p_{n}$. To perform this program in the most efficient way we develop a diagrammatic graphical method that allows us to write the renormalization equation directly.

We can associate a diagram with each process that contributes to the renormalization equation. The basic structure of these diagrams is reported in Fig. 7. The four intersections represent the sites of a cell. The energy transfer is indicated by an arrow pointing in the direction of the flow. In terms of diagrams a site relaxes if some arrow points out of the corresponding intersection. At this point, to enumerate the processes we simply have to analyze all the possible diagrams. Since the dynamics is completely isotropic the analysis can be reduced to processes starting from the upper left side of the cell.

First we have to eliminate diagrams that do not correspond to dynamical processes considered in the renormalization scheme. In practice we discard three classes of diagrams (Fig. 8).

(1) Diagrams where it is not possible to identify a connected path starting from the upper left site [Fig. 8(a)].

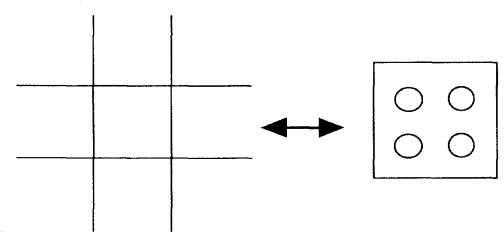

FIG. 7. The structure of the diagram associated with each cell we use in the calculation of the weight of the renormalized processes.

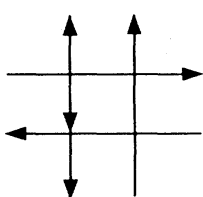

a)

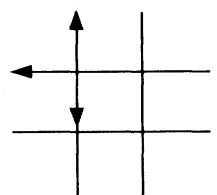

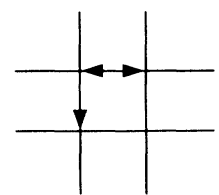

c)
FIG. 8. Three diagrams that do not contribute to the renormalization equation because of connection (a), spanning (b), and absence of energy transfer outside the cell (c).

(2) Diagrams that do not satisfy the spanning condition (i.e., with less than two relaxation processes) [Fig. 8(b)].

(3) Diagrams that do not lead to an energy transfer out of the cell [Fig. 8(c)].

We then assign a statistical weight to the effective diagrams by obeying the following rules.

(i) Each site relaxes if there is at least one arrow pointing out from it.

(ii) The number of outgoing arrows gives the statistical weight of each process:

$$
\begin{aligned}
& 1 \text { arrow } \rightarrow \frac{1}{4} p_{1}, \\
& 2 \text { arrows } \rightarrow \frac{1}{6} p_{2}, \\
& 3 \text { arrows } \rightarrow \frac{1}{4} p_{3}, \\
& 4 \text { arrows } \rightarrow p_{4} .
\end{aligned}
$$

The statistical weight of the entire diagram is given by the product of the statistical weight of each subprocess.

(iii) Each diagram contributes to the renormalization of a process at a larger scale of type $n$ given by the correspondent number of different sides of the diagram having an outgoing arrow.

(iv) Each diagram has an additional statistical weight $W_{\alpha}(\rho)$ [Eq. (9)], where $\alpha$ is the number of critical sites.

Using these rules we can write the renormalization equation in the following form:

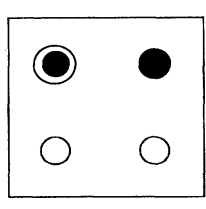

a)

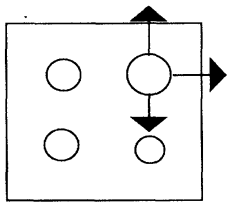

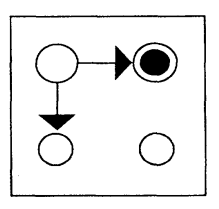

b)

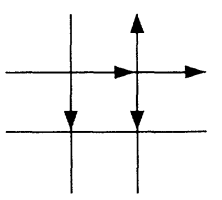

d)
FIG. 9. A process (a)-(c) that contributes to the renormalization of the dynamics and the correspondent diagram (d). 
TABLE III. Values of the nonzero polynomial term coefficients of the renormalization transformation (see text). Each value represents the weight of all the subprocesses that contribute to the renormalization equation for a given starting cell with the same polynomial form in the dynamical parameters. The coefficients are ordered with respect to the starting configuration $\alpha$.

\begin{tabular}{|c|c|c|c|c|c|c|c|c|c|c|c|}
\hline$n$ & $x_{1}$ & $x_{2}$ & $x_{3}$ & $x_{4}$ & $A$ & $n$ & $x_{1}$ & $x_{2}$ & $x_{3}$ & $x_{4}$ & $A$ \\
\hline \multicolumn{12}{|c|}{$\alpha=2$} \\
\hline 1 & 0 & 0 & 2 & 0 & $6.2500000 \times 10^{-2}$ & 2 & 1 & 1 & 0 & 1 & 0.7777781 \\
\hline 1 & 0 & 1 & 1 & 0 & 0.2916667 & 2 & 1 & 1 & 1 & 0 & 0.7083317 \\
\hline 1 & 0 & 2 & 0 & 0 & 0.2222223 & 2 & 1 & 2 & 0 & 0 & 0.2037039 \\
\hline 1 & 1 & 0 & 1 & 0 & 0.4375000 & 2 & 2 & 0 & 0 & 1 & 0.4375001 \\
\hline 1 & 1 & 1 & 0 & 0 & 0.4583334 & 2 & 2 & 0 & 1 & 0 & 0.2291666 \\
\hline 1 & 2 & 0 & 0 & 0 & 0.1250000 & 2 & 2 & 1 & 0 & 0 & $6.9444463 \times 10^{-2}$ \\
\hline 2 & 0 & 0 & 1 & 1 & 0.5000000 & 3 & 0 & 0 & 1 & 1 & 0.3333333 \\
\hline 2 & 0 & 0 & 2 & 0 & 0.3750000 & 3 & 0 & 0 & 1 & 2 & 0.5000000 \\
\hline 2 & 0 & 1 & 0 & 1 & 0.8333336 & 3 & 0 & 0 & 2 & 0 & 0.2083333 \\
\hline 2 & 0 & 1 & 1 & 0 & 0.7083332 & 3 & 0 & 0 & 2 & 1 & 0.7916664 \\
\hline 2 & 0 & 2 & 0 & 0 & 0.2222223 & 3 & 0 & 0 & 3 & 0 & 0.2708333 \\
\hline 2 & 1 & 0 & 0 & 1 & 1.000000 & 3 & 0 & 1 & 0 & 1 & 0.3333334 \\
\hline 2 & 1 & 0 & 1 & 0 & 0.5000000 & 3 & 0 & 1 & 0 & 2 & 0.8888893 \\
\hline 2 & 1 & 1 & 0 & 0 & 0.1666667 & 3 & 0 & 1 & 1 & 0 & 0.2500000 \\
\hline 3 & 0 & 0 & 0 & 2 & 1.000000 & 3 & 0 & 1 & 1 & 1 & 0.749997 \\
\hline 3 & 0 & 0 & 1 & 1 & 1.250000 & 3 & 0 & 1 & 2 & 0 & 0.6527764 \\
\hline 3 & 0 & 0 & 2 & 0 & 0.3125000 & 3 & 0 & 2 & 0 & 0 & $3.7037041 \times 10^{-2}$ \\
\hline 3 & 0 & 1 & 0 & 1 & 0.6666669 & 3 & 0 & 2 & 0 & 1 & 0.7222226 \\
\hline 3 & 0 & 1 & 1 & 0 & 0.2500000 & 3 & 0 & 2 & 1 & 0 & 0.3981479 \\
\hline 3 & 0 & 2 & 0 & 0 & $2.7777780 \times 10^{-2}$ & 3 & 0 & 3 & 0 & 0 & $5.5555552 \times 10^{-2}$ \\
\hline 3 & 1 & 0 & 0 & 1 & 0.2500000 & 3 & 1 & 0 & 0 & 1 & 0.1666667 \\
\hline \multirow[t]{4}{*}{3} & 1 & 0 & 1 & 0 & $6.2500000 \times 10^{-2}$ & 3 & 1 & 0 & 0 & 2 & 1.166667 \\
\hline & & & & & & 3 & 1 & 0 & 1 & 0 & $8.3333336 \times 10^{-2}$ \\
\hline & \multirow{2}{*}{\multicolumn{5}{|c|}{$\alpha=3$}} & 3 & 1 & 0 & 1 & 1 & 1.625001 \\
\hline & & & & & & 3 & 1 & 0 & 2 & 0 & 0.4270836 \\
\hline 1 & 0 & 1 & 1 & 0 & $5.5555560 \times 10^{-2}$ & 3 & 1 & 1 & 0 & 1 & 1.000000 \\
\hline 1 & 0 & 1 & 2 & 0 & $2.0833334 \times 10^{-2}$ & 3 & 1 & 1 & 1 & 0 & 0.3680552 \\
\hline 1 & 0 & 2 & 0 & 0 & 0.1296296 & 3 & 1 & 2 & 0 & 0 & $4.1666664 \times 10^{-2}$ \\
\hline 1 & 0 & 2 & 1 & 0 & $7.4074052 \times 10^{-2}$ & 3 & 2 & 0 & 0 & 1 & 0.2500000 \\
\hline 1 & 0 & 3 & 0 & 0 & $4.6296299 \times 10^{-2}$ & 3 & 2 & 0 & 1 & 0 & $5.203328 \times 10^{-2}$ \\
\hline 1 & 1 & 0 & 1 & 0 & 0.2083333 & 4 & 0 & 0 & 0 & 3 & 1.000000 \\
\hline 1 & 1 & 0 & 2 & 0 & $5.2083328 \times 10^{-2}$ & 4 & 0 & 0 & 1 & 2 & 2.000000 \\
\hline 1 & 1 & 1 & 0 & 0 & 0.4444445 & 4 & 0 & 0 & 2 & 1 & 1.187500 \\
\hline 1 & 1 & 1 & 1 & 0 & 0.2569445 & 4 & 0 & 0 & 3 & 0 & 0.1979166 \\
\hline 1 & 1 & 2 & 0 & 0 & 0.1712964 & 4 & 0 & 1 & 0 & 2 & 1.166667 \\
\hline 1 & 2 & 0 & 0 & 0 & 0.1666667 & 4 & 0 & 1 & 1 & 1 & 1.222222 \\
\hline 1 & 2 & 0 & 1 & 0 & 0.1770833 & 4 & 0 & 1 & 2 & 0 & 0.2500001 \\
\hline 1 & 2 & 1 & 0 & 0 & 0.1458334 & 4 & 0 & 2 & 0 & 1 & 0.2685184 \\
\hline 1 & 3 & 0 & 0 & 0 & $2.0833334 \times 10^{-2}$ & 4 & 0 & 2 & 1 & 0 & $7.8703679 \times 10^{-2}$ \\
\hline 2 & 0 & 0 & 2 & 0 & $8.3333336 \times 10^{-2}$ & 4 & 0 & 3 & 0 & 0 & $4.6296306 \times 10^{-3}$ \\
\hline 2 & 0 & 0 & 2 & 1 & $6.2500000 \times 10^{-2}$ & 4 & 1 & 0 & 0 & 2 & 0.5000000 \\
\hline 2 & 0 & 0 & 3 & 0 & $7.2916664 \times 10^{-2}$ & 4 & 1 & 0 & 1 & 1 & 0.4583334 \\
\hline 2 & 0 & 1 & 0 & 1 & 0.2222223 & 4 & 1 & 0 & 2 & 0 & $7.2916664 \times 10^{-2}$ \\
\hline 2 & 0 & 1 & 1 & 0 & 0.4166668 & 4 & 1 & 1 & 0 & 1 & 0.1666667 \\
\hline 2 & 0 & 1 & 1 & 1 & 0.3055556 & 4 & 1 & 1 & 1 & 0 & $2.7777778 \times 10^{-2}$ \\
\hline 2 & 0 & 1 & 2 & 0 & 0.3402775 & 4 & 2 & 0 & 0 & 1 & $2.0833334 \times 10^{-2}$ \\
\hline 2 & 0 & 2 & 0 & 0 & 0.2222222 & & & & & & \\
\hline 2 & 0 & 2 & 0 & 1 & 0.2870370 & & & & & & \\
\hline 2 & 0 & 2 & 1 & 0 & 0.3935182 & & & & & & \\
\hline 2 & 0 & 3 & 0 & 0 & 0.1111111 & & & & & & \\
\hline 2 & 1 & 0 & 0 & 1 & 0.5000000 & & & & & & \\
\hline 2 & 1 & 0 & 1 & 0 & 0.4583334 & & & & & & \\
\hline 2 & 1 & 0 & 1 & 1 & 0.5000001 & & & & & & \\
\hline 2 & 1 & 0 & 2 & 0 & 0.4062502 & 1 & 0 & 2 & 0 & 0 & $5.5555560 \times 10^{-2}$ \\
\hline 2 & 1 & 1 & 0 & 0 & 0.2222222 & 1 & 0 & 2 & 2 & 0 & $6.9444450 \times 10^{-3}$ \\
\hline
\end{tabular}


TABLE III. (Continued).

\begin{tabular}{|c|c|c|c|c|c|c|c|c|c|c|c|}
\hline$n$ & $x_{1}$ & $x_{2}$ & $x_{3}$ & $x_{4}$ & $A$ & $n$ & $x_{1}$ & $x_{2}$ & $x_{3}$ & $x_{4}$ & $A$ \\
\hline 1 & 0 & 3 & 1 & 0 & $2.7777772 \times 10^{-2}$ & 3 & 0 & 0 & 4 & 0 & 0.2265625 \\
\hline 1 & 0 & 4 & 0 & 0 & $2.3148149 \times 10^{-2}$ & 3 & 0 & 1 & 1 & 0 & 0.2500000 \\
\hline 1 & 1 & 1 & 0 & 0 & 0.4166666 & 3 & 0 & 1 & 1 & 1 & 0.2500000 \\
\hline 1 & 1 & 1 & 1 & 0 & $6.2500000 \times 10^{-2}$ & 3 & 0 & 1 & 1 & 2 & 1.000000 \\
\hline 1 & 1 & 1 & 2 & 0 & $4.1666668 \times 10^{-2}$ & 3 & 0 & 1 & 2 & 0 & 0.2291667 \\
\hline 1 & 1 & 2 & 0 & 0 & 0.1111111 & 3 & 0 & 1 & 2 & 1 & 2.229165 \\
\hline 1 & 1 & 2 & 1 & 0 & 0.1770832 & 3 & 0 & 1 & 3 & 0 & 1.067710 \\
\hline 1 & 1 & 3 & 0 & 0 & 0.1435186 & 3 & 0 & 2 & 0 & 0 & $5.5555560 \times 10^{-2}$ \\
\hline 1 & 2 & 0 & 0 & 0 & 0.2500000 & 3 & 0 & 2 & 0 & 1 & 0.2777778 \\
\hline 1 & 2 & 0 & 1 & 0 & 0.1562500 & 3 & 0 & 2 & 0 & 2 & 0.9444448 \\
\hline 1 & 2 & 0 & 2 & 0 & $5.4687500 \times 10^{-2}$ & 3 & 0 & 2 & 1 & 0 & 0.3750001 \\
\hline 1 & 2 & 1 & 0 & 0 & 0.2291667 & 3 & 0 & 2 & 1 & 1 & 2.902769 \\
\hline 1 & 2 & 1 & 1 & 0 & 0.3177083 & 3 & 0 & 2 & 2 & 0 & 1.597237 \\
\hline 1 & 2 & 2 & 0 & 0 & 0.2673606 & 3 & 0 & 3 & 0 & 0 & 0.1018518 \\
\hline 1 & 3 & 0 & 0 & 0 & $6.2500000 \times 10^{-2}$ & 3 & 0 & 3 & 0 & 1 & 1.046295 \\
\hline 1 & 3 & 0 & 1 & 0 & 0.1484375 & 3 & 0 & 3 & 1 & 0 & 0.8541641 \\
\hline 1 & 3 & 1 & 0 & 0 & 0.1562500 & 3 & 0 & 4 & 0 & 0 & 0.1280864 \\
\hline 1 & 4 & 0 & 0 & 0 & $1.5625000 \times 10^{-2}$ & 3 & 1 & 0 & 1 & 0 & 0.1250000 \\
\hline 2 & 0 & 0 & 4 & 0 & $7.8125000 \times 10^{-3}$ & 3 & 1 & 0 & 1 & 1 & 0.6250000 \\
\hline 2 & 0 & 1 & 1 & 0 & 0.1666667 & 3 & 1 & 0 & 1 & 2 & 1.375000 \\
\hline 2 & 0 & 1 & 2 & 1 & $4.1666668 \times 10^{-2}$ & 3 & 1 & 0 & 2 & 0 & 0.3750000 \\
\hline 2 & 0 & 1 & 3 & 0 & 0.1041666 & 3 & 1 & 0 & 2 & 1 & 2.531250 \\
\hline 2 & 0 & 2 & 0 & 0 & 0.2222222 & 3 & 1 & 0 & 3 & 0 & 1.007812 \\
\hline 2 & 0 & 2 & 0 & 1 & $8.3333343 \times 10^{-2}$ & 3 & 1 & 1 & 0 & 1 & 0.8333334 \\
\hline 2 & 0 & 2 & 1 & 0 & $9.7222239 \times 10^{-2}$ & 3 & 1 & 1 & 0 & 2 & 2.500000 \\
\hline 2 & 0 & 2 & 1 & 1 & 0.1666667 & 3 & 1 & 1 & 1 & 0 & 0.6458334 \\
\hline 2 & 0 & 2 & 2 & 0 & 0.3489575 & 3 & 1 & 1 & 1 & 1 & 5.916649 \\
\hline 2 & 0 & 3 & 0 & 0 & $9.2592582 \times 10^{-2}$ & 3 & 1 & 1 & 2 & 0 & 2.562505 \\
\hline 2 & 0 & 3 & 0 & 1 & 0.1388889 & 3 & 1 & 2 & 0 & 0 & 0.1250000 \\
\hline 2 & 0 & 3 & 1 & 0 & 0.3842584 & 3 & 1 & 2 & 0 & 1 & 2.694436 \\
\hline 2 & 0 & 4 & 0 & 0 & 0.1772839 & 3 & 1 & 2 & 1 & 0 & 1.618071 \\
\hline 2 & 1 & 0 & 1 & 0 & 0.3750000 & 3 & 1 & 3 & 0 & 0 & 0.2222220 \\
\hline 2 & 1 & 0 & 2 & 0 & $9.3750000 \times 10^{-2}$ & 3 & 2 & 0 & 0 & 1 & 0.3750000 \\
\hline 2 & 1 & 0 & 2 & 1 & 0.1250000 & 3 & 2 & 0 & 0 & 2 & 1.625000 \\
\hline 2 & 1 & 0 & 3 & 0 & 0.1875000 & 3 & 2 & 0 & 1 & 0 & 0.1562500 \\
\hline 2 & 1 & 1 & 0 & 0 & 0.3333333 & 3 & 2 & 0 & 1 & 1 & 2.750000 \\
\hline 2 & 1 & 1 & 0 & 1 & 0.4166666 & 3 & 2 & 0 & 2 & 0 & 0.8593750 \\
\hline 2 & 1 & 1 & 1 & 0 & 0.5624999 & 3 & 2 & 1 & 0 & 1 & 1.937497 \\
\hline 2 & 1 & 1 & 1 & 1 & 0.6875001 & 3 & 2 & 1 & 1 & 0 & 0.7500011 \\
\hline 2 & 1 & 1 & 2 & 0 & 1.000003 & 3 & 2 & 2 & 0 & 0 & $7.6388918 \times 10^{-2}$ \\
\hline 2 & 1 & 2 & 0 & 0 & 0.3472224 & 3 & 3 & 0 & 0 & 1 & 0.3437500 \\
\hline 2 & 1 & 2 & 0 & 1 & 0.6805550 & 3 & 3 & 0 & 1 & 0 & $6.2500000 \times 10^{-2}$ \\
\hline 2 & 1 & 2 & 1 & 0 & 1.309034 & 4 & 0 & 0 & 0 & 4 & 1.000000 \\
\hline 2 & 1 & 3 & 0 & 0 & 0.4004621 & 4 & 0 & 0 & 1 & 3 & 4.000000 \\
\hline 2 & 2 & 0 & 0 & 1 & 0.5000000 & 4 & 0 & 0 & 2 & 1 & 0.1875000 \\
\hline 2 & 2 & 0 & 1 & 0 & 0.3750000 & 4 & 0 & 0 & 2 & 2 & 5.437500 \\
\hline 2 & 2 & 0 & 1 & 1 & 0.5937500 & 4 & 0 & 0 & 3 & 0 & 0.1250000 \\
\hline 2 & 2 & 0 & 2 & 0 & 0.6093750 & 4 & 0 & 0 & 3 & 1 & 2.937500 \\
\hline 2 & 2 & 1 & 0 & 0 & 0.2083334 & 4 & 0 & 0 & 4 & 0 & 0.5156250 \\
\hline 2 & 2 & 1 & 0 & 1 & 0.9375006 & 4 & 0 & 1 & 0 & 3 & 3.833335 \\
\hline 2 & 2 & 1 & 1 & 0 & 1.192708 & 4 & 0 & 1 & 1 & 1 & 0.4166666 \\
\hline 2 & 2 & 2 & 0 & 0 & 0.3628464 & 4 & 0 & 1 & 1 & 2 & 9.416665 \\
\hline 2 & 3 & 0 & 0 & 1 & 0.3437500 & 4 & 0 & 1 & 2 & 0 & 0.2812500 \\
\hline 2 & 3 & 0 & 1 & 0 & 0.2578125 & 4 & 0 & 1 & 2 & 1 & 6.781219 \\
\hline 2 & 3 & 1 & 0 & 0 & $7.2916664 \times 10^{-2}$ & 4 & 0 & 1 & 3 & 0 & 1.359372 \\
\hline 3 & 0 & 0 & 2 & 0 & 0.1250000 & 4 & 0 & 2 & 0 & 1 & 0.2222222 \\
\hline 3 & 0 & 0 & 2 & 2 & 0.2500000 & 4 & 0 & 2 & 0 & 2 & 3.638883 \\
\hline 3 & 0 & 0 & 3 & 1 & 0.5000000 & 4 & 0 & 2 & 1 & 0 & 0.1527778 \\
\hline
\end{tabular}


TABLE III. (Continued).

\begin{tabular}{|c|c|c|c|c|c|c|c|c|c|c|c|}
\hline$n$ & $x_{1}$ & $x_{2}$ & $x_{3}$ & $x_{4}$ & $A$ & $n$ & $x_{1}$ & $x_{2}$ & $x_{3}$ & $x_{4}$ & $A$ \\
\hline 4 & 0 & 2 & 1 & 1 & 4.555559 & 4 & 1 & 1 & 1 & 0 & $8.3333328 \times 10^{-2}$ \\
\hline 4 & 0 & 2 & 2 & 0 & 1.119792 & 4 & 1 & 1 & 1 & 1 & 5.666653 \\
\hline 4 & 0 & 3 & 0 & 0 & $1.3888890 \times 10^{-2}$ & 4 & 1 & 1 & 2 & 0 & 1.104168 \\
\hline 4 & 0 & 3 & 0 & 1 & 0.8564807 & 4 & 1 & 2 & 0 & 1 & 1.291664 \\
\hline 4 & 0 & 3 & 1 & 0 & 0.3171290 & 4 & 1 & 2 & 1 & 0 & 0.3333326 \\
\hline 4 & 0 & 4 & 0 & 0 & $2.2376545 \times 10^{-2}$ & 4 & 1 & 3 & 0 & 0 & $1.6203705 \times 10^{-2}$ \\
\hline 4 & 1 & 0 & 0 & 3 & 3.500000 & 4 & 2 & 0 & 0 & 1 & $6.2500000 \times 10^{-2}$ \\
\hline 4 & 1 & 0 & 1 & 1 & 0.2500000 & 4 & 2 & 0 & 0 & 2 & 1.562500 \\
\hline 4 & 1 & 0 & 1 & 2 & 7.750000 & 4 & 2 & 0 & 1 & 1 & 1.460250 \\
\hline 4 & 1 & 0 & 2 & 0 & 0.1250000 & 4 & 2 & 0 & 2 & 0 & 0.1953125 \\
\hline 4 & 1 & 0 & 2 & 1 & 4.937500 & 4 & 2 & 1 & 0 & 1 & 0.4687498 \\
\hline 4 & 1 & 0 & 3 & 0 & 0.8359375 & 4 & 2 & 1 & 1 & 0 & $6.2500007 \times 10^{-2}$ \\
\hline 4 & 1 & 1 & 0 & 1 & 0.2500000 & 4 & 3 & 0 & 0 & 1 & $3.1250000 \times 10^{-2}$ \\
\hline 4 & 1 & 1 & 0 & 2 & 5.249998 & & & & & & \\
\hline
\end{tabular}

$$
\begin{aligned}
p_{n}^{\prime}=\sum_{\alpha} W_{\alpha}(\rho) \frac{1}{N_{\alpha}} \sum_{x_{1}, x_{2}, x_{3}, x_{4}} p_{1}^{x_{1}} p_{2}^{x_{2}} p_{3}^{x_{3}} p_{4}^{x_{4}} \\
\times A_{n}\left(\alpha, x_{1}, x_{2}, x_{3}, x_{4}\right),
\end{aligned}
$$

where $N_{\alpha}$ is a normalization constant that takes into account all the effective diagrams of each starting configuration $\alpha$, and then is a function of $\vec{P}$ in its turn. Here $A_{n}(\ldots)$ is a matrix whose elements are the sum of the weight of all the diagrams that, starting from a given cell, renormalize in the same process at a larger scale with the same polynomial form with respect to the variables $p_{i}$. As an example the diagram in Fig. 9(d) contributes to the matrix element $A_{n=2}(\alpha=2,0,1,1,0)$ with a value of $a=\frac{1}{24}$.

In Table III we report the nonzero elements of the ma$\operatorname{trix} A$.
[1] P. Bak, C. Tang, and K. Wiesenfeld, Phys. Rev. Lett. 59, 381 (1987); Phys. Rev. A 38, 364 (1988).

[2] P. Bak, K. Chen, and M. Creutz, Nature 342, 780 (1989); P. Bak, K. Chen, J. Scheinkmen, and M. Woodford, Rich. Economiche 47, 3 (1993).

[3] T. A. Witten and L. M. Sander, Phys. Rev. Lett. 47, 1400 (1981).

[4] T. Vicsek, Fractal Growth Phenomena (World Scientific, Singapore, 1992).

[5] R. Cafiero, V. Loreto, L. Pietronero, A. Vespignani, and S. Zappieri, Europhys. Lett. 29, 111 (1995).

[6] D. Sornette, J. Phys. (Paris) 1 2, 2065 (1992).

[7] P. Bak and M. Creutz, in Fractals and Disordered Systems, edited by A. Bunde and S. Havlin (Springer-Verlag, Heidelberg, 1993), Vol. II.

[8] L. P. Kadanoff, Physica A 163, 1 (1990); Phys. Today 44(3), 9 (1991).

[9] L. Pietronero, A. Erzan, and C. Evertsz, Phys. Rev. Lett. 61, 861 (1988); A. Erzan, L. Pietronero, and A. Vespignani (unpublished).

[10] R. Cafiero, L. Pietronero, and A. Vespignani, Phys. Rev. Lett. 70, 3939 (1993).

[11] L. Pietronero, A. Vespignani, and S. Zapperi, Phys. Rev. Lett. 72, 1690 (1994).

[12] S. S. Manna, L. B. Kiss, and J. Kertesz, J. Stat. Phys. 61,
923 (1990).

[13] Z. Olami, H. J. S. Feder, and K. Christensen, Phys. Rev. Lett. 68, 1244 (1992); K. Christensen and Z. Olami, Phys. Rev. A 46, 1829 (1992).

[14] Y. C. Zhang, Phys. Rev. Lett. 63, 470 (1989).

[15] L. Pietronero, P. Tartaglia, and Y. C. Zhang, Physica A 173, 129 (1991).

[16] S. S. Manna, J. Phys. A 24, L363 (1991).

[17] K. Christensen, H. C. Fogedby, and H. J. Jensen, J. Stat. Phys. 61, 653 (1991).

[18] S. S. Manna, J. Stat. Phys. 59, 509 (1990); Physica A 179, 249 (1991).

[19] L. P. Kadanoff, S. R. Nagel, L. Wu, and S. Zhu, Phys. Rev. A 39, 6524 (1989).

[20] P. Grassberger and S. S. Manna, J. Phys. (France) 51, 1077 (1990).

[21] T. Hwa and M. Kardar, Phys. Rev. Lett. 62, 1813 (1989).

[22] A. Diaz-Guilera, Phys. Rev. A 45, 8551 (1992); Europhys. Lett. 26, 177 (1994).

[23] D. Dhar and R. Ramaswamy, Phys. Rev. Lett. 63, 1659 (1989); D. Dhar, ibid. 64, 1613 (1991); S. N. Majumdar and D. Dhar, Physica A 185, 129 (1992).

[24] M. Paczuski, S. Maslov, and P. Bak, Europhys. Lett. 27, 97 (1994).

[25] T. W. Burkhard and J. M. J. Van Leuween, Real Space 
Renormalization (Springer, Heidelberg, 1982).

[26] R. J. Creswick, H. A. Farach, and C. P. Poole, Jr., Introduction to Renormalization Group Methods in Physics (Wiley, New York, 1992).

[27] C. Tang and P. Bak, Phys. Rev. Lett. 60, 2347 (1988).
[28] L. Pietronero and W. R. Schneider, Phys. Rev. Lett. 66, 2336 (1991).

[29] G. Caldarelli and R. Hallgass (private communication).

[30] V. Loreto, L. Pietronero, A. Vespignani, and S. Zapperi (unpublished). 